American Journal of Pharmaceutical Education 2019; 83 (6) Article 7442.

\title{
COMMENTARY
}

\section{Pharmacy Education Needs to Address Diagnostic Safety}

\author{
Mark L. Graber, MD, ${ }^{\mathrm{a}}$ Gloria R Grice, PharmD, ${ }^{\mathrm{b}}$ Louis J. Ling, MD, ${ }^{\mathrm{c}}$ Jeannine M. Conway, PharmD, ${ }^{\mathrm{d}}$ \\ Andrew Olson, $\mathrm{MD}^{\mathrm{e}}$ \\ ${ }^{a}$ The Society to Improve Diagnosis in Medicine, Plymouth, Massachusetts \\ ${ }^{\mathrm{b}}$ St. Louis College of Pharmacy, St. Louis, Missouri \\ ${ }^{c}$ Accreditation Council for Graduate Medical Education, Chicago, Illinois \\ ${ }^{\mathrm{d}}$ University of Minnesota, College of Pharmacy, Minneapolis, Minnesota \\ ${ }^{\mathrm{e}}$ University of Minnesota Medical School, Minneapolis, Minnesota
}

Submitted November 28, 2018; accepted February 14, 2019; published August 2019.

\begin{abstract}
The American Association of Colleges of Pharmacy, the Accreditation Council for Pharmacy Education, and the Center for the Advancement of Pharmacy Education frame patient safety from the perspective of medication management, which is also the current focus of pharmacy education and training. With the growing appreciation that diagnostic errors represent an urgent and actionable patient safety concern, the National Academy of Medicine has recommended diagnostic safety training for all health care professions. The Society to Improve Diagnosis in Medicine has worked with an interprofessional consensus group to identify a set of 12 key competencies necessary to achieve diagnostic quality and safety that focuses on individual, team-based, and system-related competencies. Much of this already exists in pharmacy education, but pharmacy training programs need to give graduates more guidance on how they contribute to the diagnostic process and the prevention and detection of diagnostic errors. We describe the current state of progress in this regard, and what steps are needed by training programs to provide content and assessment so that graduates achieve the requisite competencies. Governing and advisory bodies need to expand the expectations around patient safety to include diagnostic safety.
\end{abstract}

Keywords: Interprofessional, diagnosis, educational competency, diagnostic error, patient safety

\section{INTRODUCTION}

The profession of pharmacy has a longstanding commitment to promoting quality and safety in clinical practice. In response to the first call to improve safety from the Institute of Medicine in 1999, the American Association of Colleges of Pharmacy's (AACP) Argus Commission declared that "a culture of safety should permeate the practice of pharmacy in all settings."1,2 The 2007 Argus Report specifically recommended that colleges and schools of pharmacy include education and training on medication safety. These recommendations have been strongly endorsed and incorporated into expectations from both the Accreditation Council for Pharmacy Education (ACPE) and the Center for the Advancement of Pharmacy Education (CAPE) ${ }^{3,4}$ A series of reports in 2011 found that education programs had made substantial progress in responding to this challenge. ${ }^{5-8}$

Although medication safety remains an active problem in clinical practice, it is, unfortunately, not the only

Corresponding Author: Mark L Graber, The Society to Improve Diagnosis in Medicine, 5 Hitching Post, Plymouth, MA 02360. Tel: 919-667-8585. E-mail:

Mark.Graber@Improvediagnosis.org safety challenge confronting patients. ${ }^{9,10}$ The recent National Academy of Medicine (NAM) report "Improving Diagnosis in Healthcare" called attention to diagnostic errors as another major patient safety concern, potentially rivaling or exceeding the harm from medication-related safety events in its scope. ${ }^{11}$ According to the NAM report, diagnostic error is "likely to affect each of us in our lifetimes," and accounts for 40,000-80,000 deaths annually in the US. The report and its recommendation are directed at all health care professions and their practitioners. Specifically, the NAM report has important implications for pharmacy education. Despite the many advances the pharmacy profession has realized, ${ }^{12}$ for example in achieving advanced pharmacist practice stature and collaborative protocol legislation, the pharmacy profession still views itself as having just a supporting role in the diagnostic process.

\section{The Diagnostic Process and the Role of Pharmacists}

The NAM report describes diagnosis as a process. It begins when the patient experiences a health concern and seeks care. It includes evaluation and clinical decisionmaking leading to consideration of the diagnostic 


\section{American Journal of Pharmaceutical Education 2019; 83 (6) Article 7442.}

possibilities, and subsequent actions, including followup. Pharmacists already contribute to the process at essentially every step: they provide an access point for patients to engage with the health care system, they judge the importance of a patient's problem and triage its priority, they ask the patient thoughtful questions to understand the patient's problems, they consider appropriate explanations for those problems (diagnoses), they monitor for diagnostic error, and they promote learning from both diagnostic successes and failures. Although the scope of practice laws in most states would not permit pharmacists to diagnose illness, we believe this concept is antiquated. Pharmacists are already contributing to the diagnostic process by using their expertise. This role needs to be recognized, encouraged and included as a major focus of pharmacy education and training. The vignettes in Table 1 illustrate examples of how pharmacists contribute to the diagnostic process. Pharmacists may recognize new symptoms or signs as manifestations of drug interactions or drug toxicity, or an unsuspected medical condition. These are the kind of everyday observations pharmacists make as part of the Pharmacy Quality Alliance's IESA (indication, effectiveness, safety, adherence) medication as- sessment framework, ${ }^{13}$ perhaps without realizing their immediate connection to diagnosis and diagnostic safety.

The many ways in which pharmacists contribute to the health care team help determine the quality and safety of the diagnostic process. For example, the community pharmacist is often the first point of contact when a patient realizes they have a health care concern and seeks help. The pharmacist must immediately decide if the problem requires further evaluation at a higher level of care (eg, clinic or emergency care setting). Diagnosis is often a matter of "distributed cognition," where different team members may know a unique piece of the puzzle that is needed for successful diagnosis. Pharmacists know their patients and their medication history and can often provide critical information about medications and their health impact that relate to diagnosis. Another example of the pharmacists' role on the team is their acting as "error checkers." Fresh eyes catch mistakes, and it is easier to recognize the errors made by others than the ones we make ourselves. ${ }^{14,15}$ Pharmacists have the opportunity to see whether a new diagnosis makes sense based on what they are observing in the patient, and if errors are suspected, to intervene before there is harm. In practice,

Table 1. Vignettes Illustrating Pharmacist Contributions to Diagnosis (with IESA Classification)

\section{Individual Competency}

A patient notes the new onset of diarrhea and abdominal cramps, and asked the pharmacist where to find an over-the-counter antimotility medication. Incidentally, the patient mentioned that the diarrhea was bloody. The pharmacist recognized this to be a symptom in need of urgent investigation, and advised that the patient seek help at the Emergency Department. This resulted in the more timely diagnosis and management of ischemic colitis; serious adverse consequences would have been likely if the diagnosis had been delayed.

(IESA: Indication - unnecessarylinappropriate medication therapy)

An elderly patient notes increasing shortness of breath on exertion. The patient has a 50 pack-year-history of smoking, and still smokes a pack per day. Using a regularly-calibrated flowmeter, the pharmacist determines that the patient has severe obstruction to airflow on exhalation. Referring the patient to his primary care provider expedites the diagnosis of chronic obstructive pulmonary disease.

(IESA: Indication - Untreated condition)

\section{Team-based Competency}

A patient presents with increasing fatigue. The pharmacist notices that the patient was recently given a beta blocker by her new cardiologist, who may not have appreciated that the patient was already receiving a different beta blocker from her internist. The pharmacist contacts the internist (primary care provider), notifies her of the duplicated order, and clarifies with the patient that one beta blocker is enough.

(IESA: Safety - Adverse medication event; medication interaction)

\section{System-related Competency}

An inpatient pharmacist is processing the medications for a patient admitted with somnolence and confusion, and notes that "L4-5 disc herniation and spinal stenosis" are listed as an active problem. On accessing a regional database, the pharmacist discovers that the patient is receiving both short- and long-acting opiates from several different pharmacists and providers. The pharmacist notifies the responsible clinicians of the finding, and brings up the possibility that the patient's mental status changes may be from excessive use of narcotics.

(IESA: Safety - Dosage too high) 


\section{American Journal of Pharmaceutical Education 2019; 83 (6) Article 7442.}

effective interprofessional care would involve frequent communication with the patient's other health care providers (or empowering the patient to self-advocate) to discuss existing diagnoses and possible diagnostic alternatives. The need for re-evaluation of a given diagnosis in view of informed assessments based on the expertise and experience of the pharmacist is critical. Patient safety breakdowns are particularly evident during transitions of care, and pharmacists can identify them during medication reconciliation. Effective communication among all of the patient's clinical providers is essential at these junctures.

The diagnostic process works in most cases, but diagnostic errors are common. These errors are estimated to occur in $10 \%$ of cases in primary care settings, and some of these cases will result in patient harm. ${ }^{16}$ The complexity of the diagnostic process itself, the uncertainty that exists at every step, the inherent and myriad flaws in our health care systems, and breakdowns in clinical reasoning all contribute to the problem. ${ }^{11}$

The NAM report calls on education programs in every health care profession to "enhance health care professional training and education in the diagnostic process" and further specifies that "certification and accreditation organizations should ensure that healthcare professionals have and maintain these competencies." The NAM report concluded that changes in the education of health care professionals could have a profound impact on diagnostic safety and quality but stopped short of specifying the new competencies that would be needed. To address this, the Society to Improve Diagnosis in Medicine, supported by the Josiah Macy, Jr Foundation, convened an interprofessional consensus group (pharmacists, physicians, nurses, physician assistants, laboratorians, trainees, patients, educators, and representatives from boards and certifying agencies) to identify the key competencies necessary for health care professionals to improve diagnostic safety. The NAM recommendation to improve teamwork in diagnosis was the foundational motivation for ensuring that the competencies were interprofessional in scope. The consensus group identified 12 competency concepts that are relevant to training programs in every health care profession (Table 2$).{ }^{17}$

\section{The New Competencies for Diagnosis Align with the Existing Pharmacy Competency Expectations}

The 12 new competency concepts need to be interpreted, considered, and acted upon by every health care profession. The 12 concepts are consistent with, and complement, the existing standards and outcomes for pharmacy education already promulgated by the CAPE and ACPE (Appendix 1). The congruency reflects in large part the past and present efforts by the CAPE and ACPE to keep abreast of new conceptual innovations in health care, especially those relevant to patient safety and interprofessional training and practice. This includes incorporating recommendations from the IOM report, Health Professions Education: A Bridge to Quality, the Interprofessional Education Collaborative (IPEC), the Joint Commission of Pharmacy Practitioners Pharmacist Patient Care Process (PCPP) and the Patient Care Process for delivering Comprehensive Medication Management (CMM). ${ }^{18-21}$

\section{Pharmacy Training to Improve Diagnosis - What's Already in Progress, and What's Missing?}

The 12 competency concepts are divided into three domains: Individual competencies (I-1 through I-6) are focused on the foundational skills that each member of the health care team needs to understand the patient's problems and derive an appropriate differential diagnosis; Team-based competencies (T-1 through T-3) are focused on partnering effectively with other members of the health care team, including patients, to determine the diagnostic plan; and System-related competencies (S-1 through S-3) relate to understanding the (health) system factors that can facilitate or hinder optimal diagnostic performance. We consider the current state of pharmacy education relative to each of these three domains, identifying progress already realized in each area, and what elements and actions are missing.

Individual competencies focus on how professionals use clinical reasoning to consider appropriate, justifiable, and timely diagnoses. Although pharmacists work within a defined scope of practice that does not typically include specific mention of diagnosis, the "Assess" step of the Pharmacist Patient Care Process specifically expects pharmacists to analyze collected information to "identify and prioritize problems and achieve optimal care." 20 Additionally, the AACP Core Entrustable Professional Activities specifically calls for competency in several diagnosis-related skills, including patient assessment, making appropriate triage decisions, interpreting laboratory tests, and compiling a prioritized health-related problem list. ${ }^{22}$ Pharmacists already contribute to the diagnostic process, following the IESA guidance, including recognizing new symptoms or signs as manifestations of drug interactions or drug toxicity, or an unsuspected medical condition.

Advanced pharmacist practice models are expanding. In this context, point-of-care testing is increasingly used to assist with diagnosis. ${ }^{23,24}$ Pharmacy-based blood pressure measurement has been used to identify patients with hypertension, and pharmacist screening can identify atrial fibrillation in high-risk patients. ${ }^{25,26}$ Additionally, new point-of-care tests have been used to identify specific infectious diseases, including viral hepatitis and HIV infection. ${ }^{27-30}$ 


\section{American Journal of Pharmaceutical Education 2019; 83 (6) Article 7442.}

Table 2. Individual, Team-based, and System-related Competencies to Improve Diagnosis

Individual Competencies: Demonstrate clinical reasoning to arrive at a justifiable diagnosis (an explanation for a healthrelated condition)

I-1. Accurately and efficiently collect key clinical findings needed to inform diagnostic hypotheses. Use these tools appropriately and efficiently in the diagnostic process: Effective interpersonal communication skills, history-taking, the physical examination, and record review; diagnostic testing; and the electronic health record and health IT resources.

I-2. Formulate, or contribute to, an accurate problem representation expressed in a concise summary statement that includes essential epidemiological, clinical, and psychosocial information.

I-3. Produce, or contribute to, a correctly prioritized, relevant differential diagnosis, including can't miss diagnoses.

I-4. Explain and justify the prioritization of the differential diagnosis by comparing and contrasting the patient's findings and test results with accurate knowledge about prototypical or characteristic disease manifestations and atypical presentations, and considering pathophysiology, disease likelihood, and clinical experience.

I-5. Use decision support tools, including point-of-care resources, checklists, consultation, and second opinions to improve diagnostic accuracy and timeliness.

I-6. Use reflection, surveillance, and critical thinking to improve diagnostic performance and mitigate detrimental cognitive bias throughout the clinical encounter. Discuss and reflect on the strengths and weaknesses of cognition, the impact of contextual factors on diagnosis, and the challenges of uncertainty. Demonstrate awareness of atypical presentations, information that is missing, and key findings that don't 'fit.'

Team-based Competencies: Partner effectively as part of an interprofessional diagnostic team. Communicate effectively and solicit information from all members of the team (including the patient and family) to create a shared mental model of a patient's illness and the plan for diagnostic evaluation.

T-1. Engage and collaborate with patients and families, in accordance with their values and preferences when making a plan for diagnostic evaluation. Listen actively, encourage questions, and be alert to new or changing information. Explain the diagnostic process, including the patient's and family's role in helping to identify the most likely diagnosis. Share appropriately when diagnostic uncertainty exists.

T-2. Collaborate with other health care professionals (including nurses, physicians, physician assistants, radiologists, laboratory professionals, pharmacists, social workers, physical therapists, medical librarians, and others) and communicate effectively throughout the diagnostic process. Acknowledge and challenge authority gradients, especially between clinicians and patients/ families, constructively.

T-3. Apply effective strategies at transitions of care to facilitate accurate and sufficient information transfer about the diagnosis, including any pending workup and areas of uncertainty. Close the loop on test result communication and clarify expectations with the team for test result follow-up.

System-related Competencies: Identify and understand the systems factors that facilitate and contribute to timely, accurate diagnoses and error avoidance.

S-1. Discuss how human factors contribute to diagnostic safety and error by identifying how the work environment influences human performance. Take steps to mitigate common systems factors that detract from diagnostic quality and safety. Use local resources (including people, teams and technology, especially the electronic health record) effectively and efficiently to optimize patients' access to care, diagnostic testing services, and appropriate experts for consultation.

S-2. Advance a culture of diagnostic safety that encourages open dialogue and continuous learning from analysis and discussion of excellent diagnostic performance, near misses and errors. Give and receive feedback at an individual and team level to improve subsequent diagnostic performance.

S-3. Disclose diagnostic errors and missed opportunities transparently and in a timely manner to patients, families, team members, supervisors, and appropriate quality and risk management staff.

Spirometry testing aids in recognizing and managing asthma and chronic obstructive pulmonary disease. ${ }^{31-33}$

Colleges and schools of pharmacy have already described and implemented content and coursework on the principles of clinical reasoning. ${ }^{34,35}$ Pharmacy accreditation standards have endorsed the importance of this training: "Graduates should be able to independently identify and solve problems using critical thinking," and recom- mendations and tools to assess critical thinking have been published. ${ }^{36-38}$

Competency in using information technology has become a de facto requirement for all health care professionals. Informatics training for student pharmacists should prepare them not only to meet needs regarding medication safety, but also to participate effectively in the diagnostic process and communicate with other 


\section{American Journal of Pharmaceutical Education 2019; 83 (6) Article 7442.}

members of the health care team. ${ }^{39}$ Clauson and colleagues, however, found that only one in three pharmacy programs included intentional informatics training, and in half of these programs, informatics training was an elective course. ${ }^{40}$

\section{Individual Competencies - What's Missing?}

Most critically, what is missing is an explicit recognition in the education standards of the role pharmacists can and should play in diagnosis. Besides their role in triage, it is appropriate for pharmacists to contribute to making diagnoses for a specified range of conditions. Further work is needed to more clearly delineate these conditions and the contexts in which pharmacists contribute to their diagnosis. Pharmacists can and should contribute to the diagnostic process and work collaboratively with other clinicians. Conversely, missing in the training of other health care professionals is the recognition of the key role of pharmacists in the diagnostic process, especially the identification and evaluation of new healthrelated problems. Interprofessional experiences during education and training could help physicians, nurses, and other health professionals appreciate how pharmacists can contribute to both diagnosis and the detection of diagnostic errors.

It is likely that training programs already include content on clinical decision-making within existing courses, but we do not know to what extent pharmacy colleges and schools do or do not incorporate this content or specific training on clinical reasoning, the derivation of a differential diagnosis, or the use of decision-support tools to assist in these processes. While pharmacists are not expected to formally list a differential diagnosis for a patient, the process of thinking through the various possible explanations of a given symptom or sign, even in strict relation to drug treatment, employs the same cognitive skillset. There are many web-accessible tools now available to help clinicians and patients formulate an appropriate differential diagnosis for a given set of key findings. ${ }^{41,42}$ Intentional incorporation of such decisionsupport tools during education and training would allow pharmacists in practice to use these tools when appropriate.

Fundamentally, the pharmacist's thought process of identifying medication therapy problems by assessing indication, effectiveness, safety, and adherence (IESA) is itself a diagnostic reasoning process. ${ }^{15,16}$ It would be worthwhile for the education community to standardize this training to improve consistency of delivery of patient care services. Clinical reasoning and critical thinking are general skills; the same coursework could equally apply to physician and nursing trainees, as well as student phar- macists. Ideally, these courses would be offered in interprofessional venues, using realistic case scenarios and simulations.

\section{Team-based Competencies}

These competencies address the recommendation to improve teamwork to improve diagnostic performance. Historically, diagnosis was often considered to be solely the responsibility of the physician, but the NAM report calls for a new, revised vision, which explicitly seeks greater involvement from everyone who "touches" the patient, including the pharmacist. The pharmacist has multiple roles on the diagnostic team, as discussed above, and a great deal of progress has already been achieved in training programs in terms of working toward interprofessional, team-based competency. Interprofessional practice is already strongly endorsed by PPCP and ACPE, supported by the competency recommendations developed by IPEC. The ACPE envisions that PharmD graduates will be "team ready." As a result, interprofessional training in colleges and schools of pharmacy has flourished in the US and internationally. ${ }^{43-49}$ Descriptions of courses on care transitions are emerging, and studies of pharmacist involvement at care transitions have shown improvements in patient care parameters and opportunities for clinical collaboration. ${ }^{50-52}$ High-fidelity simulation is a practical and highly effective approach to teach skills in interprofessional collaboration. ${ }^{53}$ Colleges and schools also have access to a wide range of tools to evaluate interprofessional education and proficiency, ${ }^{54}$ including novel objective, structured, clinical examination (OSCE)-based formats to evaluate team-based decisionmaking. ${ }^{55}$

\section{Team-based Competencies - What's Missing?}

Interprofessional experiences during pharmacy training should include standardized experiences that specifically focus on diagnosis. The vignettes in Table 1, for example, would be appropriate learning exercises. Additional ways to evaluate team-based assessment, focused specifically on diagnosis-related skills, would also be valuable.

\section{System-Related Competencies}

Focus on the idea that the timeliness and accuracy of diagnosis could be improved by appreciating the systemrelated aspects of the diagnostic process. Professional training typically focuses on acquiring professionspecific knowledge, with little emphasis on how to actually use one's knowledge effectively in unique systems. Over half of diagnostic errors involve system-related flaws, such as breakdowns in communication and care 


\section{American Journal of Pharmaceutical Education 2019; 83 (6) Article 7442.}

coordination. ${ }^{56}$ The system-related competencies emphasize why these happen and how to help patients navigate the health care system effectively and safely. Additionally, these competencies call attention to how and when systems can contribute to error and the need for all health care professionals to continually work to identify and fix system vulnerabilities. Lastly, these competencies ensure graduates appreciate the value of learning to improve safety through feedback and performance improvement and contribute to a culture of safety. Pharmacy training may be a role model in this regard, to the extent that education and training matches the ACPE expectation that graduates should be "practice ready."

As outlined earlier, the AACP, ACPE, and CAPE have all strongly endorsed patient safety as a core value. Courses designed to teach concepts of safety and quality have been described. ${ }^{57}$ Courses emphasizing "human factor" concepts are particularly important in regard to acquiring system-related competencies, as these elements explain the relationships between the tasks, the team members, and the work environment that ultimately determines the quality and safety of the diagnostic process. $^{58,59}$ Coursework on error disclosure is also valuable and recommended by NAM, but few professions include content to achieve this competency and fewer still do this in an interprofessional manner. ${ }^{60}$

\section{System-related Competencies - What's Missing?}

In the existing $\mathrm{AACP}, \mathrm{ACPE}$, and CAPE documents, safety is framed in relation to medication safety, and this has been the focus of pharmacy education and training. ${ }^{8,61}$ Our goal is to improve diagnostic safety, which will require that the governing and advisory bodies expand their vision of safety to include all aspects of patient safety, including diagnostic safety in particular. Ideally this would include education on both the cognitive- and system-related contributions to both successful and unsuccessful diagnosis. Learning to conduct (or contribute to) a root cause analysis of a diagnostic error is an excellent way to illustrate these concepts. Pharmacy graduates should have an appreciation for how they contribute to diagnostic safety, and how they can help avoid both cognitive and system-related predispositions to diagnostic error.

\section{CONCLUSION}

We have presented the case that pharmacists are important members of the diagnostic team. Depending on their training, defined scope of responsibilities and practice context, pharmacists are already acting in this capacity daily. What is missing in both the competency expectations and in pharmacy education is explicit acknowledgement of the contribution that pharmacists make in the diagnostic process, encouragement for them to participate in this process proactively and regularly, and education and training experiences that prepare them appropriately for these roles. The language of the professional competency expectations needs to reflect proper acknowledgement of diagnostic quality and safety. Training programs need to translate these expectations into relevant coursework and experiences. Ideally, this training would be done in interprofessional settings in the classroom, simulations, and clinical practice experiences.

Promoting and achieving competency in diagnosis is particularly important right now, as pharmacists are practicing in an expanding range of health care settings, and the profession is seeking "more advanced direct patient care roles." 12 Considering the cost of diagnostic errors both in dollars and in harm, the NAM asserted that "improving the diagnostic process is not only possible, but it also represents a moral, professional, and public health imperative." 11 It is critical for pharmacy educators to join with all other health care educators to address this need as quickly as possible.

\section{REFERENCES}

1. Nahata M, Beck D, Draugalis J, Flynn A, Kerr R, Wells B. The academy's agenda for improving the safety of medication use: report of the 2006-2007 argus commission. Am J Pharm Educ. 2007;

71(6):Article S18.

2. Kohn L, Corrigan J, Donaldson M. To Err is Human: Building a Safery Health System. Washington, DC: Institute of Medicine Committee on Quality of Health Care in America; 2000.

3. Medina M, Plaza C, Stowe C, et al. Center for the Advancement of Pharmacy Education 2013 Educational Outcomes. Am J Pharm Educ. 2013;77(8):Article 162.

4. American Council Pharmaceutical Education. Accreditation standards and key elements for the professional program in pharmacy leading to the Doctor of Pharmacy degree (Standards 2016). https:// www.acpe-accredit.org/pdf/Standards2016FINAL.pdf. Published February 2015. Accessed June 28, 2019.

5. Holdford DA, Warholak TL, West-Strum D, Bentley JP, Malone DC, Murphy JE. Teaching the science of safety in US colleges and schools of pharmacy. Am J Pharm Educ. 2011;75(4):Article 77. 6. Vogt E, Robinson D, Chambers-Fox S. Educating for safety in the pharmacy curriculum. Am J Pharm Educ. 2011;75(7):Article 140. 7. West-Strum D, Basak R, Bentley J, et al. The science of safety curriculum in US colleges and schools of pharmacy. Am J Pharm Educ. 2011;75(7):Article 141.

8. Tang DH, Warholak TL, Slack MK, Malone DC, Gau C-S. Science of safety topic coverage in experiential education in US and Taiwan colleges and schools of pharmacy. Am J Pharm Educ. 2011;75(10): Article 202.

9. Tache SV, Sonnichsen A, Ashcroft DM. Prevalence of adverse drug events in ambulatory care: a systematic review. Ann

Pharmacother. 2011;45(7-8):977-989. 


\section{American Journal of Pharmaceutical Education 2019; 83 (6) Article 7442.}

10. Lainer M, Vogele A, Wensing M, Sonnichsen A. Improving medication safety in primary care. A review and consensus procedure by the LINNEAUS collaboration on patient safety in primary care. Eur J Gen Pract. 2015;21 Suppl:14-18.

11. Improving Diagnosis in Health Care. National Academy of Medicine. National Academies Press, Washington, DC. 2015. 12. Knoer S, Eck A, Lucas A. A review of American pharmacy: education, training, technology, and practice. J Pharm Health Care Sci. 2016;2:32.

13. Pharmacy Quality Alliance. Medication Therapy Problem Categories Framework for PQA Measures. https://www.pqaalliance. org/pqa-measures. Accessed February 12, 2019.

14. Croskerry $P$. The importance of cognitive errors in diagnosis and strategies to minimize them. Acad Med. 2003;78(8):775-780.

15. Croskerry P. A universal model of diagnostic reasoning. Acad Med. 2009;84(8):1022-1028.

16. Graber M. The incidence of diagnostic error. BMJ Quality and Safety. 2013;22, Part 2:ii21-ii27.

17. Olson A, Cosby K, Rencic J, et al. Competencies for improving diagnosis: an interprofessional framework for education and training in healthcare. Diagnosis. 2019. https://doi.org/10.1515/dx-2018-0107 18. Institute of Medicine Committee on the Health Professions Education Summit. Health Professions Education: A Bridge to Quality. Washington, DC. 2003.

19. Interprofessional Education Collaborative. Interprofessional Collaborative Practice: IPEC Core Competencies. https://www. ipecollaborative.org/resources.html. Updated 2016. Accessed February 12, 2019.

20. Joint Commision of Pharmacy Practitioners. Pharmacists' Patient Care Process. https://jcpp.net/patient-care-process/. Accessed February 12, 2019.

21. CMM in Primary Care Research Team. The Patient Care Process for Delivering Comprehensive Medication Management (CMM):

Optimizing Medication Use in Patient-Centered Team-Based Care Settings. https://www.accp.com/cmm_care_process. Accessed February 12, 2019.

22. Haines S, Pittenger A, Stolte S, et al. Core entrustable professional activities for new pharmacy graduates. Am J Pharm Educ. 2017;81(1):Article S2.

23. Akinwale T, Adams A, Dering-Anderson A, Klepser D. Pharmacy-based point-of-care testing for infectious diseases: considerations for the pharmacy curriclum. Curr Pharm Educ Learn. 2015;7(1):131-136.

24. Bacci JL, Klepser D, Tilley H, Smith JK, Klepser ME. Community pharmacy-based point-of-care testing: A case study of pharmacist-physician collaborative working relationships. Res Social Adm Pharm. 2018;14(1):112-115.

25. Sabater-Hernandez D, Sendra-Lillo J, Jimenez-Monleon J, et al. Identifying masked uncontrolled hypertension in the community pharmacy setting. Blood Press Monit. 2015;20(3):138-143.

26. Walker N, Doughty R, Parag V, Harrison J, Bennett M, Freedman B. Pharmacy-based screening for atrial fibrillation in high-risk Maori and Pacific populations. N Z Med J. 2014;127(1398):128-131. 27. Gubbins PO, Klepser ME, Dering-Anderson AM, et al. Point-ofcare testing for infectious diseases: opportunities, barriers, and considerations in community pharmacy. J Am Pharm Assoc (2003). 2014;54(2):163-171.

28. Calderon Y, Cowan E, Schramm C, et al. HCV and HBV testing acceptability and knowledge among urban emergency department patients and pharmacy clients. Prev Med. 2014;61:29-33.
29. Isho N, Kachlic M, Marcelo J, Martin M. Pharmacist-initiated hepatitis $\mathrm{C}$ virus screening in a community pharmacy to increase awareness and link to care at the medical center. J Am Pharm Assoc. (2003). 2017;57(3S):S259-S264.

30. Amesty S, Crawford N, Nandi V, et al. Evaluation of pharmacybased HIV testing in a high-risk New York City community. AIDS Patient Care STDS. 2015;29(8):437-444.

31. Cawley MJ, Moon J, Reinhold J, Willey VJ, Warning Ii WJ. Spirometry: tool for pharmacy practitioners to expand direct patient care services. J Am Pharm Assoc (2003). 2013;53(3):307-315.

32. Senna G, Caminati M, Bovo C, Canonica G, Passalacqua G. The role of the pharmacy in the management of bronchial asthma: A literature-based evaluation. Ann Allergy Asthma Immunol.

2017;118(2):161-165.

33. Fathima M, Saini B, Foster J, Armour C. Community pharmacybased case finding for COPD in urban and rural settings is feasible and effective. Int J Chron Obstruct Pulmon Dis. 2017;12:2753-2761. 34. Nolt V, Cain J, Wermeling D. Design and delivery of a new clinical reasoning course. Curr Pharm Educ Learn. 2018;10(8):11131123.

35. Tietze K. Clinical reasoning model for pharmacy students. The Clinical Teacher. 2018;15:1-5.

36. Frenzel J, Eukel H, Neville M, et al. Essential skills for pharmacy graduates reported by advanced pharmacy practice experience preceptors. Curr Pharm Educ Learn. 2015;7(5):700-704.

37. James D, Hartzler M, Chen A. Assessment of critical thinking skills progression in a pre-pharmacy curriculum. Curr Pharm Educ Learn. 2016;8(6):767-773.

38. Gleason B, Gaebelein C, Grice G, et al. Assessment of students' critical-thinking and problem-solving abilities across a 6-year doctor of pharmacy program. Am J Pharm Educ. 2013;77(8):166.

39. Steckler T, Brownlee M, Urick B, Farley M. Pharmacy informatics: a call to action for educators, administrators, and residency directors. Curr Pharm Educ Learn. 2017;9(5):746-749. 40. Clauson K, Breedon E, Fingado A, Kaing C, Flynn A, Cutler T. A progress report on the state of pharmacy informatics education in US pharmacy schools and colleges. Am J Pharm Educ. 2018;82(7):Article 6332.

41. Bond W, Schwartz L, Weaver K, Levick D, Giuliano M, Graber M. Differential diagnosis generators: An evaluation of currently available computer programs. J Gen Int Med. 2011;27(2):213-219. 42. Riches N, Panagioti M, Alam R, et al. The effectiveness of electronic differential diagnosis (DDX) generators: A systematic review and meta-analysis. PloS one. 2016;11(3):e0148991.

43. Boland D, Scott M, Kim H, White T, Adams E. Interprofessional immersion: Use of interprofessional education collaborative competencies in side-by-side training of family medicine, pharmacy, nursing, and counselling psychology trainees. $J$ Interprof Care. 2016;30(6):739-746.

44. Rotz M, Duenas G, Zanoni A, Grover A. Designing and Evaluating an Interprofessional Experiential Course Series Involving Medical and Pharmacy Students. Am J Pharm Educ. 2016;80(5):85. 45. Hess R, Hagemeier N, Blackwelder R, Rose D, Ansari N, Branham $\mathrm{T}$. Teaching communication skills to medical and pharmacy students through a blended learning course. Am J Pharm Educ. 2016;80(4):64.

46. Peeters M, Sexton M, Metz A, Hasbrouck C. A team-based interprofessional education course for first-year health professions students. Curr Pharm Educ Learn. 2017;9(6):1099-1110. 


\section{American Journal of Pharmaceutical Education 2019; 83 (6) Article 7442.}

47. Patel N, Begum S, Kayyali R. Interprofessional Education (IPE) and Pharmacy in the UK. A Study on IPE Activities across Different Schools of Pharmacy. Pharmacy (Basel). 2016;4(4).

48. Wilson AJ, Palmer L, Levett-Jones T, Gilligan C, Outram S. Interprofessional collaborative practice for medication safety: nursing, pharmacy, and medical graduates' experiences and perspectives. J Interprof Care. 2016;30(5):649-654.

49. Lapkin S, Levett-Jones T, Gilligan C. A cross-sectional survey examining the extent to which interprofessional education is used to teach nursing, pharmacy and medical students in Australian and New Zealand universities. J Interprof Care. 2012;26(5):390-396. 50. Sen S, Hadley D, Patterson B, Patel R. Development and implementation of a transitions of care elective course. Curr Pharm Educ Learn. 2016;8(2):380-390.

51. Vavra K, Paluzzi M, de Voest M, Raguckas S, Slot M. Preliminary findings from a student pharmacist operated transitions of care pilot service. Curr Pharm Educ Learn. 2018;10(1):78-84. 52. Vogler C, Arnoldi J, Moose H, Hingle ST. Interprofessional education involving medical and pharmacy students during transitions of care. J Interprof Care. 2017;31(3):404-406.

53. Meyer B, Seefeldt $T$, Ngorsuraches $S$, et al. Interprofessional education in pharmacology using high-fidelity simulation. Curr Pharm Educ Learn. 2017;9(6):1055-1062.

54. Shrader S, Farland M, Danielson J, Sicat B, Umland E. A systematic review of assessment tools measuring interprofessional education outcomes relevant to pharmacy education. Am J Pharm Educ. 2017;81(6):Article 119.

55. Dagenais R, Pawluk S, Rainkie D, Wilby K. Team-based decision-making in an objective structured clinical examination. Innovat in Pharm. 2018;9(3):Article 14.

56. Graber ML, Franklin N, Gordon R. Diagnostic error in internal medicine. Arch Intern Med. 2005;165(13):1493-1499.

57. Lavigne J. Implementing "Educating Pharmacy Students and Pharmacists to Improve Quality" (EPIC) as a requirement at the Wegmans School of Pharmacy. Curr Pharm Educ Learn. 2012;4(3):212-216.

58. Vosper H, Hignett S. A UK perspective on human factors and patient safety education in pharmacy curricula. Am J Pharm Educ. 2018;82(3):Article 6184

59. Al Juffali L, Al-Aqeel S, Knapp P, Mearns K, Family H, Watson M. Using the Human Factors Framework to understand the origins of medication safety problems in community pharmacy in Saudi Arabia: A qualitative study. Res Social Adm Pharm. https:// doi.org/10.1016/j.sapharm.2018.07.010 Accessed February 12, 2019.

60. Ragucci K, Kern D, Shrader S. Evaluation of interprofessional team disclosure of a medical error to a simulated patient. Am J Pharm Educ. 2016;80(8):Article 138.

61. Cooley J, Stolpe S, Montoya A, et al. An analysis of quality improvement education at US colleges of pharmacy. Am J Pharm Educ. 2017;81(3):51. 


\section{American Journal of Pharmaceutical Education 2019; 83 (6) Article 7442.}

Appendix 1. Crosswalk Between the 12 New Competency Concepts and the Existing CAPE, ${ }^{a}$ EPA, ${ }^{b}$ PCP, ${ }^{c}$ and ACPE ${ }^{d}$ Expectations

a. Medina M, Plaza C, Stowe C, et al. Center for the Advancement of Pharmacy Education 2013 Educational Outcomes. Am J Pharm Educ. 2013;77(8).

b. Haines S, Pittenger A, Stolte S, et al. Core Entrustable Professional Activities for New Pharmacy Graduates. Am J Pharm Educ. 2017;81(1):S2.

c. Pharmacists' Patient Care Process. Joint Commission of Pharmacy Practitioners. https://jcpp.net/patient-care-process/. Accessed February 11, 2019.

d. Accreditation Council for Pharmacy Education. Accreditation Standards and Key Elements for Professional Program in Pharmacy Leading to the Doctor of Pharmacy Degree. Chicago, IL. 2016.

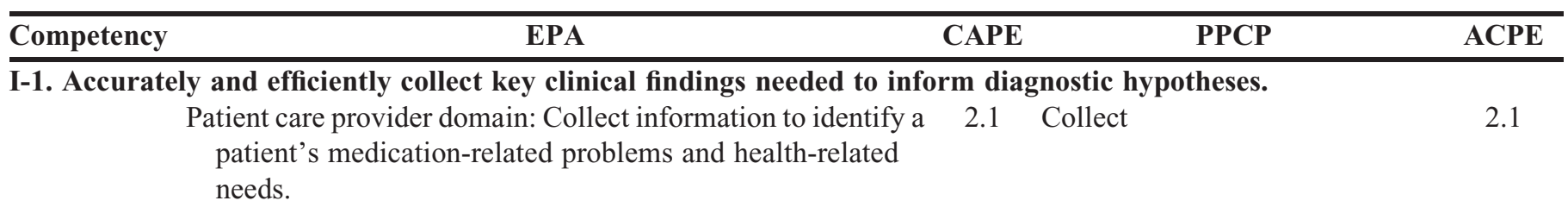

I-2. Formulate, or contribute to, an accurate problem representation expressed in a concise summary statement that includes essential epidemiological, clinical, and psychosocial information.
Patient care provider domain: Analyze information to $\quad 2.1$ Assess 2.1 determine the effects of medication therapy, identify medication-related problems, and prioritize health-related needs.

I-3. Produce, or contribute to, a correctly prioritized, relevant differential diagnosis, including can't miss diagnoses.

$\begin{array}{cccc}\text { Patient care provider domain: Analyze information to } & 2.1, & \text { Assess } & 2.1,3.1 \\ \text { determine the effects of medication therapy, identify } & 3.1 & \end{array}$
medication-related problems, and prioritize health-related needs.

I-4.Explain and justify the prioritization of the differential diagnosis by comparing and contrasting the patient's findings and test results with accurate knowledge about prototypical or characteristic disease manifestations and atypical presentations, and considering pathophysiology, disease likelihood, and clinical experience.
Patient care provider domain: Analyze information to
2.1 Assess
determine the effects of medication therapy, identify
medication-related problems, and prioritize health-related
needs.

I-5. Use decision support tools, including point-of-care resources, checklists, consultation, and second opinions to improve diagnostic accuracy and timeliness.
Patient care provider domain: Analyze information to determine the effects of medication therapy, identify
2.2 Assess, Plan medication-related problems, and prioritize health-related needs AND Implement a care plan in collaboration with the patient, caregivers, and other health professionals.

I-6. Use reflection, surveillance, and critical thinking to improve diagnostic performance and mitigate detrimental cognitive bias throughout the clinical encounter.
Self-developer domain: Create a written plan for continuous $\quad 3.1$, Collect, assess, plan, $\quad 3.1,4.1$ professional development.
4.1 implement, Follow-up:
Monitor and Evaluate

T-1.Engage and collaborate with patients and families, in accordance with their values and preferences when making a plan for diagnostic evaluation.
Patient care provider domain: Implement a care plan in
3.3, Plan
$3.3,3.4$
collaboration with the patient, caregivers, and other health
3.4
professionals. 
American Journal of Pharmaceutical Education 2019; 83 (6) Article 7442.

(Continued)

Competency

EPA

CAPE

PPCP

ACPE

T-2. Collaborate with other healthcare professionals (including nurses, physicians, physician assistants, radiologists, laboratory professionals, pharmacists, social workers, physical therapists, medical librarians, and others) and communicate effectively throughout the diagnostic process. Acknowledge and challenge authority gradients, especially between clinicians and patientslfamilies, constructively.
Interprofessional team member domain: Collaborate as a
3.3, Collect, assess, plan,
$3.3,3.4$ member of an interprofessional team.
3.4 implement, Follow-up:
11.3

Monitor and Evaluate

T-3.Apply effective strategies at transitions of care to facilitate accurate and sufficient information transfer about the diagnosis, including any pending workup and areas of uncertainty. Close the loop on test result communication and clarify expectations with the team for test result follow-up.
Patient care provider domain: Implement a care plan in $\quad 2.2$, Implement, Follow-up: $\quad$ 2.2, 3.4, collaboration with the patient, caregivers, and other health $3.4 \quad$ Monitor and Evaluate $\quad 11.3$ professionals. AND Follow-and monitor a care plan.

S-1.Discuss how human factors contribute to diagnostic safety and error by identifying how the work environment influences human performance. Take steps to mitigate common systems factors that detract from diagnostic quality and safety.
Practice manager domain: Fulfill a medication order AND
2.2 Collect, assess, plan, implement, follow-up: Oversee the pharmacy operations for an assigned work Monitor and Evaluate
2.2 appendix shift; Population Health Promoter domain: minimize adverse drug events and medication errors

S-2. Advance a culture of diagnostic safety that encourages open dialogue and continuous learning from analysis and discussion of excellent diagnostic performance, near misses and errors.

Population Health Promoter domain: minimize adverse drug events and medication errors
2.2, Collect, assess, plan, implement, follow-up: Monitor and Evaluate 2.2, 3.6, appendix 1

S-3. Disclose diagnostic errors and missed opportunities transparently and in a timely manner to patients, families, team members, supervisors, and appropriate quality and risk management staff.

\begin{tabular}{|c|c|c|c|}
\hline $\begin{array}{l}\text { Population Health Promoter domain: minimize adverse drug } \\
\text { events and medication errors }\end{array}$ & 3.6 & $\begin{array}{l}\text { Collect, assess, plan, } \\
\text { implement, follow-up: } \\
\text { Monitor and Evaluate }\end{array}$ & $\begin{array}{c}3.6, \\
\text { appendix } \\
1\end{array}$ \\
\hline
\end{tabular}

